Juliana Midori Hayashide ${ }^{a}$

José Tarcísio Penteado Buschinelli ${ }^{a}$

a Fundacentro, Programa de Pós-Graduação em Trabalho, Saúde e Ambiente. São Paulo, SP, Brasil.

Contato:

Juliana Midori Hayashide

E-mail:

juhayashide@yahoo.com.br

Os autores declaram não haver conflitos de interesses e que o trabalho não foi subvencionado.

O estudo é baseado em dissertação de mestrado de Juliana Midori Hayashide intitulada "Proposta de utilização de critérios de decisão na elaboração de protocolos de exames médicos ocupacionais para atividades críticas: o exemplo do trabalho em altura“, defendida em 2015 no Programa de Pós-Graduação Trabalho, Saúde e Ambiente, da Fundacentro. São Paulo, SP, Brasil.

O estudo não foi apresentado em reunião científica.

\section{Critérios de decisão para a definição de exames médicos ocupacionais em atividades críticas: proposição de modelo e exemplos de aplicação no trabalho em altura}

\author{
Criteria for reaching decisions on the definition of occupational \\ medical exams in critical activities: model proposition and \\ examples of application for working at height
}

\section{Resumo}

Introdução: no Brasil, tem-se observado uma grande disparidade entre os protocolos de exames médicos utilizados para avaliar trabalhadores que exercem atividades críticas. Objetivo: propor e verificar a aplicabilidade de um modelo baseado em critérios para a tomada de decisão na definição de exames médicos ocupacionais na avaliação de aptidão de trabalhadores que executam atividades críticas de trabalho. Métodos: ensaio baseado em pesquisa bibliográfica. O modelo proposto baseia-se no princípio As Low As Reasonably Practicable (ALARP), do Health and Safety Executive (HSE). O modelo foi aplicado a duas condições clínicas que podem resultar em eventos agudos de perda de consciência e levar à queda durante o trabalho em altura, caso falhem ou não tenham sido adotadas medidas de segurança: diabetes e epilepsia. Discussão: verificou-se que o modelo proposto se mostrou aplicável para os dois exemplos analisados, oferecendo subsídios para a tomada de decisão do médico do trabalho em relação à necessidade de se investigar condições clínicas que podem ocasionar acidentes fatais durante a realização de atividades críticas de trabalho.

Palavras-chave: tomada de decisões; exames médicos; gestão de riscos; saúde do trabalhador; acidentes de trabalho.

\begin{abstract}
Introduction: in Brazil, there is a considerable disparity between medical examination protocols used to evaluate workers who carry out critical activities. Objective: to propose and verify the applicability of a model based on criteria for reaching decisions on the definition of occupational medical exams that assess the capacity of workers engaged in critical activities. Methods: essay based on bibliographic research. The proposed model is based on the ALARP (As Low As Reasonably Practicable) principle of the Health and Safety Executive (HSE). The model was applied to two clinical conditions that can result in acute events of loss of consciousness and lead to falls during work at height, if no security measure was adopted: diabetes and epilepsy. Discussion: our results showed that the proposed model was applicable for both examples analyzed, offering subsidies for the occupational physician to decide on the need to investigate clinical conditions that may lead to fatal accidents during the performance of critical activities.
\end{abstract}

Keywords: decision-making; medical examination; risk management; occupational health; occupational accidents. 


\section{Introdução}

O termo "atividades críticas de trabalho" é usualmente utilizado em padrões corporativos para estabelecer requisitos e uniformidade na execução de tais tarefas. Refere-se àquelas atividades laborais com risco de acidentes fatais, para as quais o profissional necessita de qualificação específica e exigências de saúde para executar, mantendo sua integridade física, protegendo a sua saúde e preservando a vida de outras pessoas. O termo abrange atividades como: trabalho em altura, em espaço confinado, com instalações elétricas, com porte de arma de fogo; com movimentação de cargas; operadores de equipamentos móveis; condutores de veículos automotores; entre outras ${ }^{1,2}$. Neste ensaio optou-se por abordar somente o trabalho em altura.

No Brasil, de acordo com a Norma Regulamentadora $\mathrm{n}^{\circ} 35$ (NR35), trabalho em altura é considerado qualquer trabalho executado com diferença de nível superior a dois metros da superfície de referência e no qual haja risco de queda ${ }^{3}$. No Reino Unido, de acordo com o Health and Safety Executive (HSE) ${ }^{4}$, o trabalho em altura significa trabalhar em qualquer lugar onde haja diferença de nível e de onde o trabalhador possa cair de uma altura capaz de causar lesão, incluindo um lugar igual ou inferior ao nível do chão. Isso inclui também o trabalho em local que o acesso ou a saída possam levar à queda.

Diferentemente do Brasil, os regulamentos do HSE e da Occupational Safety and Health Administration (OSHA) não especificam a dimensão da diferença de nível que deve existir para que o trabalho seja considerado em altura. A OSHA define apenas que em trabalhos executados a mais de $1,8 \mathrm{~m}$, os trabalhadores sempre devem estar protegidos de quedas e de objetos em queda por equipamentos de proteção fornecidos pelo empregador ${ }^{5}$. Já no Canadá, a determinação é: quando o trabalhador apresenta risco de queda de três metros ou mais, deverá utilizar equipamentos adequados de proteção contra quedas ${ }^{6}$.

Acidentes relacionados ao trabalho em altura, principalmente em decorrência de quedas de nível, são potencialmente muito danosos e frequentemente citados como uma das principais causas de acidentes fatais, implicando custos sociais e econômicos ${ }^{7,8}$.

O planejamento do trabalho é primordial, e devem ser adotadas medidas para evitar o trabalho em altura sempre que existir meio alternativo de execução. Na impossibilidade de que se trabalhe de outra forma, devem-se adotar medidas que eliminem o risco de queda dos trabalhadores por meio de proteção coletiva, como, por exemplo, sistema de guarda-corpo e corrimãos. Quando o risco de queda não puder ser eliminado, medidas que minimizem as consequências da queda devem ser adotadas, como redes de proteção e cintos de segurança ${ }^{9}$.
A fim de reduzir o risco de quedas de altura para um nível que seja tão baixo quanto razoavelmente praticável, as análises de risco tornam-se imprescindíveis para identificar medidas de controle de riscos e de prevenção adequadas.

A análise de risco deve preceder o trabalho e contemplar todas as etapas, elementos e sequências de operações que compõem uma determinada atividade a ser realizada pelo trabalhador. Isso deve ser feito para identificar riscos potenciais de acidentes físicos e materiais, identificação e correção dos problemas operacionais e implementação da maneira correta para execução de cada etapa de trabalho com segurança ${ }^{10}$.

A atenção à saúde dos trabalhadores que executam atividades em altura é essencial porque incapacidades súbitas ou mesmo fatais durante a realização delas podem comprometer a margem de segurança necessária para evitar acidentes de trabalho. Dessa maneira, as situações de saúde que se apresentam sem sintomas premonitórios ou com sintomas súbitos e intensamente debilitantes podem colocar em risco a vida do próprio trabalhador, dos demais trabalhadores a seu redor e, dependendo da atividade realizada, até mesmo da população residente próxima às instalações de trabalho, além de causar danos ao patrimônio da empresa.

Existem diversos fatores de saúde que podem levar a acidentes durante essas atividades; algumas condições físicas, clínicas e psíquicas podem originar mal súbito ou prejudicar o desempenho funcional do trabalhador. Porém, não existe consenso em relação ao que deve ser investigado para evitar ou minimizar essas ocorrências, como essa investigação deve proceder, se exames complementares devem ser realizados e quais seriam esses exames.

Assim, com intuito de colaborar para essa discussão, este ensaio teve como objetivo propor e verificar a aplicabilidade de um modelo baseado em critérios para a tomada de decisão na definição de exames médicos ocupacionais na avaliação de aptidão de trabalhadores que executam atividades críticas de trabalho. Para verificar a aplicabilidade do modelo proposto, o método foi aplicado a duas condições clínicas que podem ocasionar eventos agudos de saúde e acarretar acidentes durante o trabalho em altura caso falhem ou não tenham sido adotadas medidas de segurança: diabetes mellitus (DM) e epilepsia.

\section{Métodos}

Este ensaio foi baseado em pesquisa bibliográfica. Para a coleta de dados, foi realizado levantamento de artigos indexados nas bases de dados SciELO, Web of Science, LILACS e Capes, em um primeiro momento 
sem delimitação do período temporal. Dentre os artigos encontrados, os mais recentes e considerados de maior relevância serviram, em uma segunda etapa, como indicação de outras fontes, por meio de suas referências. Procurou-se priorizar as fontes mais recentes, de 2004 a 2015, em línguas inglesa e portuguesa, sem descartar fontes consideradas relevantes anteriores a 2004 .

Para propor um modelo de decisão que resultasse na elaboração de um protocolo de exames médicos ocupacionais para atividades críticas, optou-se por partir do referencial teórico utilizado pelo $\mathrm{HSE}^{11}$ sobre tolerabilidade dos riscos e o princípio As Low As Reasonably Practicable (ALARP), que será posteriormente descrito, e tomando como base algumas etapas do seu sistema de abordagem para tomada de decisões.

O modelo proposto consiste de três etapas:

- Etapa 1: caracterização do evento agudo de saúde;

- Etapa 2: caracterização do evento agudo de saúde em termos de riscos e tomada de decisões;

- Etapa 3: avaliação das opções disponíveis para o gerenciamento de riscos no âmbito dos exames médicos ocupacionais.

Para demonstrar a utilização e exemplificar o modelo de tomada de decisão proposto, ele foi aplicado em duas condições clínicas que podem culminar em eventos agudos que levam à instabilidade postural e a acidentes quando ocorridos durante o trabalho em altura, caso falhem ou não tenham sido adotadas medidas de segurança: diabetes mellitus (DM), culminando em hipoglicemia, e epilepsia, com crise convulsiva.

Para as etapas 1 e 3, foi necessário realizar revisão de literatura, como descrito:

\section{Etapa 1: Caracterização do evento agudo de saúde}

As questões norteadoras dessa etapa da revisão de literatura foram: a) Qual a prevalência da patologia a ser estudada na população em geral? e b) Qual a frequência com que o evento agudo de saúde ocorre?

Para aplicar o modelo proposto nos casos de diabetes e epilepsia, utilizaram-se as seguintes palavras-chave para responder à questão a: "diabetes" × "prevalência”; "epilepsia” $\times$ "prevalência”; e os seguintes termos na língua inglesa: "diabetes" $\times$ "prevalence"; "epilepsy” $\times$ "prevalence".

Foram selecionados 47 artigos sobre diabetes, sendo que para elaboração deste ensaio foram utilizados três, de acordo com os seguintes critérios: para diabetes tipo 1 (DM1) e tipo 2 (DM2), optou-se por utilizar o estudo do International Diabetes Federation ${ }^{12}$ por representar um valor mundial, não apenas de um país. Para os diabéticos em tratamento com insulina, utilizou-se o estudo de Virally ${ }^{13}$ por apresentar uma taxa de prevalência maior entre os estudos selecionados, uma vez que se pretende abranger mais pessoas, como proteção. Para os diabéticos em tratamento com sulfonilureia, exclusivamente, foi utilizado o estudo de Brouwer ${ }^{14}$ por fornecer o dado referente ao uso exclusivo desta medicação.

Em relação à epilepsia, foram selecionados nove artigos e um foi utilizado, tendo-se optado pelo estudo de Fernandes et al. ${ }^{15}$ por englobar doença ativa e apresentar uma maior prevalência de epilepsia na população em geral, uma vez que se pretende abranger mais pessoas como proteção.

Para a questão b foram utilizados os termos “diabetes" × “complicação", “diabetes" × "hipoglicemia”, “epilepsia” × “complicação”, “epilepsia” $\times$ "crises convulsivas"; na língua inglesa, "diabetes" × "complications", "diabetes" $\times$ "hypoglycemia", "epilepsy” × “complications", "epilepsy” × "seizure”.

Referente a diabetes, foram selecionados dezoito artigos e utilizados dois. O critério de inclusão consistiu em selecionar os artigos que forneciam a frequência de ocorrência do evento agudo de saúde na forma de episódios a cada paciente por ano (episódios/paciente/ano). Assim, para DM1 e DM2, optou-se por utilizar o estudo do UK Hypoglycaemia Study Group $^{16}$. Para DM1, essa opção se deve ao estudo apresentar a maior frequência de hipoglicemia encontrada, permitindo que o pior cenário seja considerado, e também por trabalhar com uma população em que a idade e o tempo de doença se assemelham aos de um trabalhador adulto. Em relação ao DM2 em tratamento com insulina, optou-se por utilizar a frequência encontrada em diabéticos tipo 2, em insulinoterapia há mais de 5 anos, do mesmo estudo $^{16}$, por ser a maior frequência e para que fosse considerado o pior cenário. Para os diabéticos em tratamento com sulfonilureia, exclusivamente, foi utilizado o estudo de Schopman et al. ${ }^{17}$ por fornecer o dado referente ao uso exclusivo dessa medicação.

Para epilepsia, foram selecionados dezesseis artigos e utilizado um. Optou-se por utilizar o estudo de Takeda et al. ${ }^{18}$ por possuir maior amostra e por apresentar crises convulsivas decorrentes exclusivamente de epilepsia. Foram excluídos os artigos que se referiam a doentes graves, com epilepsia refratária ou de difícil controle, ou hospitalizados ou com doença neurológica e/ou psiquiátrica subjacente, por não retratarem a realidade do dia a dia do médico do trabalho, ainda mais quando se trata de uma avaliação de aptidão para atividades críticas. 
Etapa 3: avaliação das opções disponíveis para o gerenciamento de riscos

As seguintes questões foram tomadas como base: c) O rastreamento dessas moléstias, em uma população assintomática, traz benefícios no diagnóstico ou prevenção de complicações de tais patologias? e d) Existem danos associados a esse método de rastreamento?

Para a aplicação do modelo proposto em diabetes e epilepsia, foram utilizadas as seguintes palavras-chave para a questão c: "diabetes" $\times$ "rastreamento", "diabetes" $\times$ "diagnóstico", "glicemia de jejum" $\times$ "acurácia”, "glicemia de jejum" $\times$ "validade dos testes", "epilepsia" $\times$ "rastreamento", "epilepsia" $\times$ "diagnóstico", "eletroencefalografia" $\times$ "acurácia", "eletroencefalografia" $\times$ "validade dos testes", "diabetes" × "mass screening", "diabetes" × "diagnosis", "fasting blood glucose" $\times$ "accuracy", "fasting blood glucose" $\times$ "validity of tests", "epilepsy" × "mass screening", "epilepsy" × "diagnosis", "electroencephalography" × "accuracy", "electroencephalography" $\times$ "validity of tests". Foram selecionados 42 artigos sobre diabetes e utilizados sete na elaboração dessa parte do ensaio ${ }^{19-25}$. Para epilepsia, foram selecionados 35 artigos e utilizados onze ${ }^{26-36}$. Devido à limitação de tamanho permitido na publicação deste ensaio, foram utilizados os artigos mais recentes ou representativos de sociedades.

Para a questão d, as palavras-chave foram: "glicemia de jejum" $\times$ "erros de diagnóstico"; "glicemia de jejum" $\times$ "reações falso-positivas ou negativas"; "eletroencefalografia" $\times$ "erros de diagnóstico"; "eletroencefalografia" $\times$ "reações falso-positivas ou negativas"; "fasting blood glucose" $\times$ "diagnostic errors"; "fasting blood glucose" × "false-positive or false-negative reactions"; "electroencephalography" $\times$ "diagnostic errors"; "electroencephalography" $\times$ "false-positive or false-negative reactions". Sobre diabetes, foram selecionados nove artigos e utilizado um na elaboração deste ensaio ${ }^{37}$. Sobre epilepsia, foram selecionados 22 e utilizados seis $28,29,31,34-36$. Devido à limitação de tamanho permitido na publicação deste ensaio, foram utilizados os artigos mais recentes ou representativos de sociedades.

Para o desenvolvimento da Etapa 2 do modelo, utilizaram-se as bases do princípio ALARP $^{11} \mathrm{e}$ informações obtidas na literatura a respeito da prevalência das doenças consideradas e da probabilidade esperada de ocorrência dos eventos agudos de saúde potencialmente causadores de acidentes. Esses aspectos e suas aplicações serão detalhados adiante.

O projeto não foi submetido a Comitê de Ética em Pesquisa por não envolver investigações em seres humanos, atendimento clínico ou aplicação de entrevistas e questionários e por utilizar apenas fontes de informações secundárias disponíveis ao público em geral.

\section{Critérios para a tomada de decisões: o princípio ALARP}

A avaliação de risco busca analisar um evento gerador de possíveis acidentes - avaliados pela frequência, probabilidade e possível consequência; posteriormente, são feitas comparações com critérios previamente definidos para avaliação quanto a sua tolerabilidade e, caso necessário, definidas medidas a serem implantadas para a sua redução. Dessa maneira, fornece uma compreensão dos riscos, suas causas, consequências e probabilidades, o que fornecerá subsídios para decidir sobre a gestão e tratamento desses riscos ${ }^{11,38,39}$.

Segundo a ISO Guide 73, critérios de risco são valores de referência (normas, medidas ou expectativas) utilizados para interpretar as estimativas de risco obtidas por avaliações quantitativas, com o propósito de decidir o significado do risco avaliado. $\mathrm{Na}$ avaliação de risco, a frequência ou probabilidade de ocorrência de acidentes podem ser comparados com esses critérios previamente definidos para avaliação quanto a seu grau de risco. Os critérios de risco podem ser baseados em objetivos organizacionais, contextos internos e externos, padrões, leis, políticas, níveis históricos de riscos relacionados ao trabalho ou ainda outros requisitos ${ }^{40}$.

Uma das aplicações mais antigas registradas sobre tomada de decisão a respeito de riscos estava relacionada ao risco financeiro na indústria de seguro marítimo, em meados de 1700 na Inglaterra. Em seguida, evoluiu para a avaliação de riscos tecnológicos, como na construção de diques a partir de 1953 na Holanda; na indústria aeroespacial, por meio de um trabalho pioneiro de avaliação quantitativa do risco aplicado ao sistema de controle de lançamento de mísseis e espaçonaves em 1960 e nas indústrias nucleares em 1975, até chegar às aplicações atuais, nos diversos processos industriais. Os métodos e modelos de avaliação do risco mudaram com o tempo, refletindo riscos cada vez mais complexos e mudanças na ciência e tecnologia ${ }^{41}$.

Atualmente, diversos países e instituições utilizam o sistema de abordagem à tomada de decisões sobre o risco, como Reino Unido, Holanda, Suíça, Noruega, Hungria, Hong Kong, Singapura, Malásia, Austrália, Department of Defense (EUA) e International Maritime Organization (EUA ${ }^{41}$.

Optou-se por aplicar neste ensaio o referencial teórico utilizado pelo HSE no Reino Unido por ser 
um país com grande tradição na área de saúde e segurança do trabalho, e porque o referencial foi elaborado especificamente para os riscos ocupacionais.

Os critérios que o HSE desenvolveu e utiliza para tomar decisões quanto aos riscos de uma atividade ou processo é conhecido como "tolerabilidade do risco" (tolerability of risk - TOR), ou o princípio ALARP. Esses critérios podem, em teoria, ser aplicados a todos os perigos. A escolha de medidas para controlar o risco será boa o suficiente dependendo de onde os limites estiverem estabelecidos: se entre as regiões inaceitável, tolerável ou amplamente aceitável ${ }^{11}$.

O critério, tolerabilidade dos riscos, adotado pelo HSE é representado por um desenho esquemático com divisão em três regiões (Figura 1). O triângulo representa o aumento do nível de risco para uma atividade perigosa em particular (medido pela magnitude do risco individual e da preocupação da sociedade) a medida que se avança da parte inferior do triângulo para o alto. As regiões são divididas da seguinte maneira ${ }^{11,38,42}$ :

a) Uma região superior, na qual a magnitude do risco é considerada inaceitável, independente do benefício que a atividade possa trazer. A redução do risco é indispensável. Qualquer atividade que resulte em risco classificado nesta região não deve ser realizada, a não ser que as medidas de redução de risco sejam aplicadas de modo que a magnitude do risco residual caia para uma das regiões inferiores. b) Uma região intermediária, chamada de região ALARP ou região de tolerabilidade, na qual a magnitude dos riscos residuais, após estabelecidas medidas de controle, não é demasiadamente elevada e é mantida tão baixa quanto razoavelmente praticável (ALARP). Significa que os esforços para reduzir a magnitude dos riscos devem ser continuados até que o custo das medidas necessárias para tal redução sejam razoáveis quando comparados aos benefícios obtidos em termos de redução de riscos.

c) Uma região inferior denominada amplamente aceitável, na qual a magnitude do risco é considerada insignificante e adequadamente controlável, ou tão pequena que nenhuma medida de tratamento é necessária. Os níveis de risco que caracterizam essa região são comparáveis aos que as pessoas consideram como insignificante ou trivial em suas vidas diárias.

Essas três regiões são delimitadas por duas linhas horizontais, representadas pelo critério superior de risco (risco máximo tolerável) e pelo critério inferior de risco (risco amplamente aceitável). O HSE considera o critério superior de risco, ou risco máximo tolerável individual para trabalhadores, como sendo de 1 acidente fatal em $10^{3}$ trabalhadores por ano, e o critério inferior de risco, ou risco amplamente aceitável individual para trabalhadores, de 1 acidente fatal em $10^{6}$ trabalhadores por ano (Figura 1).

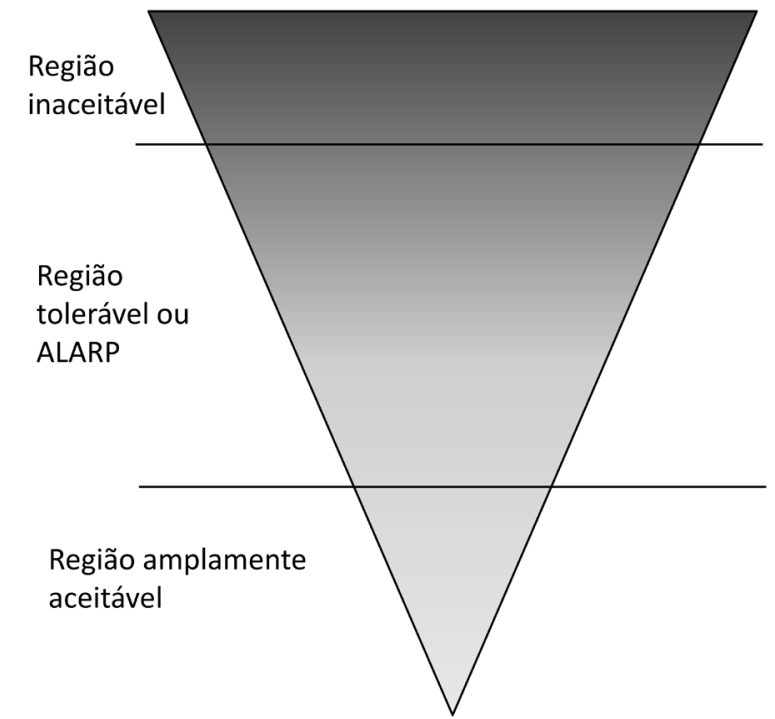

Risco insignificante
Critério superior de risco = risco máximo tolerável $=$ 1 acidente fatal em $10^{3}$ trabalhadores/ano

Critério inferior de risco $=$ risco amplamente aceitável $=1$ acidente fatal em $10^{6}$ trabalhadores/ano

Figura 1 Níveis de tolerabilidade de risco no princípio ALARP Fonte: Adaptado de Health and Safety Executive ${ }^{10,41}$ 
A tolerância ao risco não tem o mesmo significado que a aceitação do risco. É difícil que outros indivíduos ou organizações possam impor às pessoas um nível aceitável de potencial de dano. Entretanto, enquanto as pessoas não consideram essa situação aceitável, pode existir um nível de risco a que elas considerem tolerável se submeter em troca de um benefício que essa exposição acarrete. Exposições ao risco decorrentes do trabalho, do menor custo de produção, da conveniência pessoal ou da manutenção de infraestrutura social geralmente são tolerados pela sociedade. Afinal, todos vivem cercados de diversos tipos de riscos em suas atividades diárias, mas estão dispostos a tolerar muitos deles devido aos benefícios que lhes trazem, como a utilização de gás e eletricidade ou a utilização de aviões para viagens. Essa tolerância pode ser baseada, em partes, na confiança de que os esforços para reduzir os riscos são contínuos $^{11,41}$.

É importante salientar que a Figura 1 é um modelo conceitual, uma vez que os fatores e processos que contribuem para decidir se o risco é inaceitável, tolerável ou amplamente aceitável são de natureza dinâmica e, por vezes, regidos por circunstâncias particulares, como o tempo e o ambiente. Os padrões mudam, as expectativas do público mudam e o que é inaceitável em uma sociedade pode ser tolerável em outra, e o que é tolerável pode diferir em momentos de paz ou de guerra ${ }^{11}$.

O HSE ${ }^{11}$ adota como critério de risco individual a probabilidade de ocorrência de acidente fatal por ano de exposição de um trabalhador ou indivíduo localizado a certa distância da fonte. Esse acidente fatal pode decorrer de um acidente na referida instalação industrial ou evento agudo de saúde seguido de morte. Já o risco social refere-se ao risco de ocorrência de acidente fatal de toda uma comunidade exposta, em decorrência de uma operação anormal de uma instalação ou qualquer atividade humana. Indica o potencial de ocorrência de acidentes fatais com múltiplas vítimas. Como o foco deste ensaio é o trabalhador, será adotado como referencial o risco individual para trabalhadores.

O HSE acredita que um risco individual de morte de um em um milhão por ano para os trabalhadores e para indivíduos da população em geral corresponde a um nível muito baixo de risco e deve ser usado como parâmetro para o limite entre as regiões amplamente aceitáveis e toleráveis. Esse parâmetro é denominado de critério de risco amplamente aceitável. Já o limite entre as regiões inaceitável e tolerável representa um risco individual de morte de 1 em 1.000 por ano para os trabalhadores e de um em 10 mil por ano para indivíduos da população em geral (Figura 1), sendo considerado como critério de risco máximo tolerável ${ }^{11,42}$.
Para estipular esse número do critério de risco individual máximo tolerável para trabalhadores, o HSE baseou-se em observações de que esse seria o maior risco observado (e tolerado) entre as indústrias de maior risco, como a pesca submarina ${ }^{41}$.

Na prática, o HSE ${ }^{11}$ considera que a taxa de mortalidade real para os trabalhadores, mesmo nos setores mais perigosos, é normalmente bem abaixo desse limite superior estabelecido (limite máximo tolerável). Por exemplo, entre os anos de 1999 e 2000, a taxa de mortalidade anual para a agricultura, caça, silvicultura e pesca foi de 1 em 12.984; para a construção, 1 em 21.438; e para a indústria extrativa (incluindo a off-shore de petróleo e gás), 1 em 14.564, todas numericamente bem menores do que $1 \mathrm{em}$ 1.000. Em setores tradicionalmente menos perigosos, o risco anual de morte para os trabalhadores é ainda menor; por exemplo, no mesmo período para o setor de serviços, a taxa foi de 1 em 388.565, evidenciando que esse critério de risco é seguro e que existe uma margem de segurança ${ }^{11}$.

\section{Proposta de modelo de tomada de decisão para avaliação de trabalhadores que executam atividades críticas}

O modelo aqui proposto baseia-se em critérios de decisão e fornece subsídios na definição de exames médicos ocupacionais para avaliação admissional de trabalhadores que executam atividades críticas. Esse modelo de tomada de decisão classifica os eventos de saúde quanto ao seu grau de risco e forma de controle. Conforme mencionado em Métodos, consiste de três etapas baseadas no sistema de abordagem para tomada de decisões do $\mathrm{HSE}^{11}$ :

- Etapa 1: caracterização do evento agudo de saúde;

- Etapa 2: caracterização do evento agudo de saúde em termos de riscos e tomada de decisões;

- Etapa 3: avaliação das opções disponíveis para o gerenciamento de riscos no âmbito dos exames médicos ocupacionais.

A Figura 2 demonstra o modelo de tomada de decisão que está sendo aqui proposto, aplicável para os diversos eventos de saúde que podem levar a acidentes durante a realização de atividades críticas.

Na etapa 1, a caracterização do evento agudo de saúde é obtida pelo conhecimento técnico e científico de fatores - condições clínicas, físicas e psíquicas - que podem originar mal súbito ou prejudicar o desempenho funcional do trabalhador. Uma vez identificados esses fatores, é preciso conhecer suas 


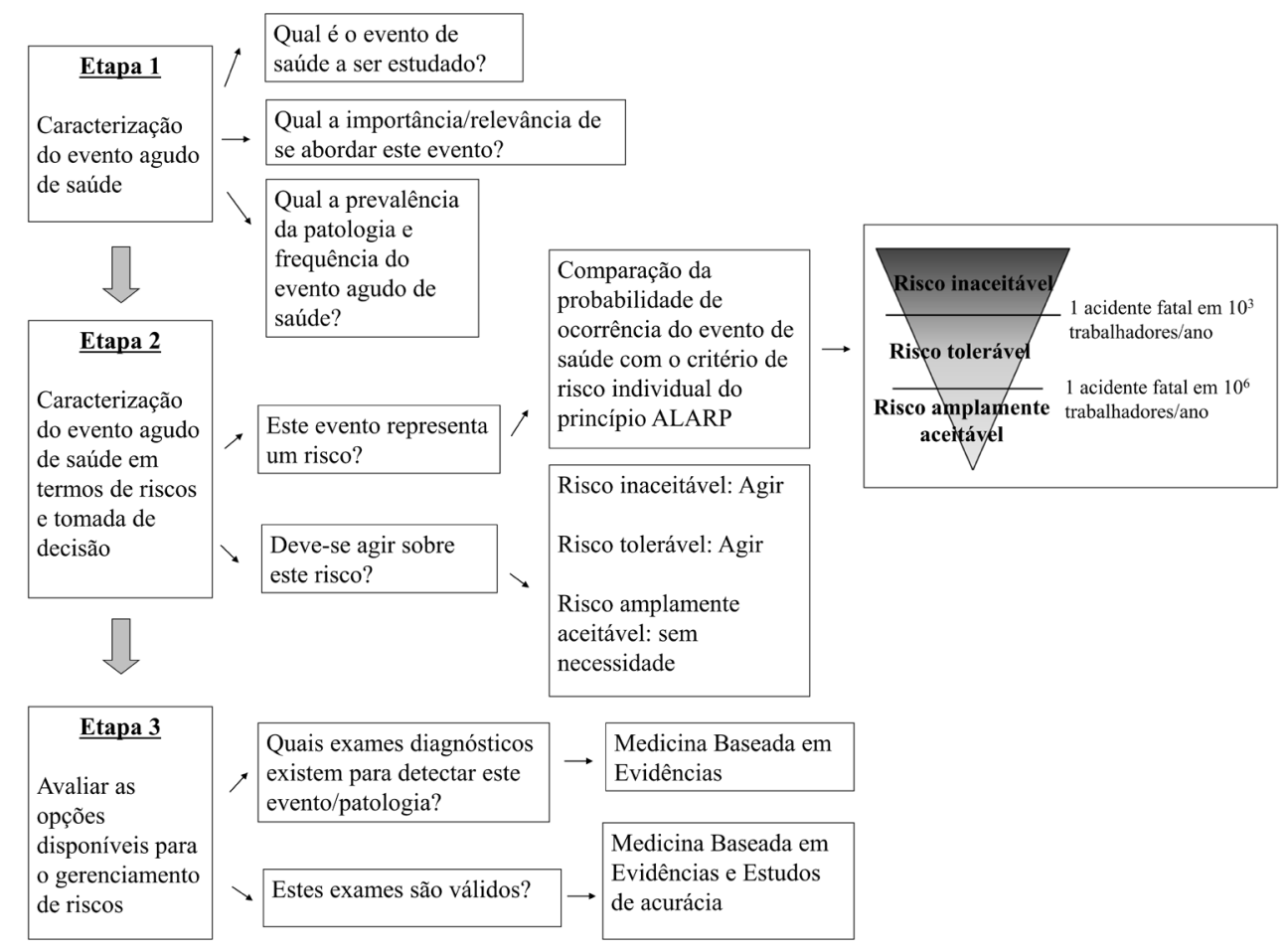

Figura 2 Modelo de tomada de decisão para a definição de exames médicos ocupacionais de avaliação de trabalhadores que executam atividades críticas

dimensões, características clínicas e epidemiológicas e relevância na população em geral.

Conhecer a prevalência das doenças auxilia os médicos a priorizar as possibilidades diagnósticas durante uma avaliação médica. Além disso, a prevalência da doença afeta fortemente a interpretação dos testes diagnósticos. Assim, para saber quais patologias investigar durante uma avaliação admissional, em quais intervir - e como intervir - para alterar o curso de um desfecho, é preciso basear-se na epidemiologia e na medicina baseada em evidências.

Na etapa 2, para a caracterizar o evento agudo de saúde em termos de riscos e tomada de decisões, é preciso conhecer a frequência desse evento agudo entre os acometidos pela condição clínica em questão e a prevalência desta na população em geral. A probabilidade do evento que pode levar à queda do trabalhador será, então, obtida pelo produto da prevalência da doença pela frequência de episódios de eventos agudos (episódios/paciente/ano). Existe alto potencial de que os acidentes ocorridos durante as atividades críticas de trabalho sejam fatais, e essa probabilidade calculada será considerada equivalente à probabilidade de ocorrer um acidente fatal. Sabe-se que nem todo acidente nessa condição será fatal; porém, para maximizar a proteção à saúde do trabalhador, deve-se sempre considerar o pior cenário.
O significado dessa probabilidade de ocorrência do evento agudo de saúde quando analisado isoladamente não faz sentido, pois sem comparação com um parâmetro, não é possível saber se o valor é baixo ou alto. Assim, propõe-se comparar essa probabilidade com os valores dos limites de tolerabilidade adotados pelo HSE, do princípio ALARP, tomando como referência o valor do critério de risco individual máximo tolerável para trabalhadores - de 1 acidente fatal em 1.000 trabalhadores por ano - e o critério de risco individual amplamente aceitável - de 1 acidente fatal em 1 milhão de trabalhadores por ano. Por essa comparação, os eventos agudos de saúde em análise são classificados em uma das três faixas da tolerabilidade de riscos (Figura 1), sendo, então, classificados quanto ao seu grau de risco como inaceitável, tolerável ou amplamente aceitável.

Após essa classificação, torna-se necessário tomar uma decisão sobre o que fazer com os eventos agudos de saúde em análise uma vez definido se são forem considerados riscos inaceitáveis, toleráveis ou amplamente aceitáveis. Deve-se decidir quando intervir para evitar ou diminuir as ocorrências e, consequentemente, para que o risco de um acidente fatal seja minimizado. A redução do risco de acidentes se dá primordialmente por medidas organizacionais e de segurança do trabalho. Porém, a avaliação médica ocupacional também pode contribuir para essa finalidade por meio do rastreamento de eventos agudos 
que podem provocar acidentes graves e fatais ou da investigação com testes diagnósticos para identificação desses agravos.

Assim, pelo princípio ALARP, o gerenciamento de riscos pode variar de acordo com as faixas do esquema de tolerabilidade. No caso da avaliação médica ocupacional, quando a probabilidade de ocorrência do evento agudo de saúde em análise se encontra na faixa amplamente aceitável, pode-se considerar que não há necessidade de adotar medidas preventivas, como a realização de testes diagnósticos. Porém, quando se encontra nas faixas tolerável ou inaceitável, indica a necessidade de reduzi-la a níveis inferiores, por exemplo, pelo rastreamento do evento agudo de saúde em questão.

Na etapa 3, uma vez definidos quais eventos de saúde devem ser abordados a fim de diminuir o risco de acidentes fatais, deve-se avaliar os meios necessários para rastreá-los na avaliação ocupacional. Deve-se considerar que se trata de uma avaliação ocupacional em que grande parte da população é assintomática. Nesses casos, o rastreamento dessas moléstias deve trazer benefícios para o diagnóstico ou a prevenção de descompensação de tais patologias e não deve provocar danos às pessoas avaliadas. Deve-se, também, avaliar se o teste diagnóstico é eficaz em termos de sensibilidade, especificidade, valores preditivos positivo e negativo, acurácia e questões como simplicidade, custo, segurança e aceitabilidade.

Quando se pensa em teste diagnóstico, geralmente se imagina um teste realizado em um laboratório, mas é importante que se tenha em mente que os princípios de validade desses testes se aplicam igualmente às informações clínicas obtidas pela anamnese, pelo exame físico e por procedimentos de imagem ${ }^{43}$. Assim, as informações que a anamnese e o exame físico fornecem também têm valor preditivo. Desta forma, quando se realiza uma boa avaliação clínica, aumenta-se a utilidade diagnóstica dos sinais clínicos e aumenta-se o valor preditivo positivo dos exames a serem solicitados, ou seja, quando o paciente é bem avaliado clinicamente, o poder diagnóstico dos exames complementares aumenta ${ }^{44}$.

A validade de um teste refere-se a quanto, em termos quantitativos ou qualitativos, um teste é útil para diagnosticar um evento ou para predizê-lo. O teste diagnóstico ideal deveria fornecer, sempre, a resposta correta, ou seja, um resultado positivo na presença da doença e um resultado negativo na ausência da doença ${ }^{45}$.

Diariamente, os médicos lidam com incertezas, ainda que não estejam cientes disso. Dessa maneira, para que possam evoluir em sua prática, tentar quantificar suas incertezas e tomar decisões apropriadas, é importante que desenvolvam conhecimentos de epidemiologia, de metodologia científica e de bioestatística ${ }^{46}$.

Exames de monitoração biológica, de rastreamento, diagnósticos e outras investigações devem ser escolhidos levando-se em consideração a validade e relevância em proteger a saúde do trabalhador, de acordo com a sensibilidade, especificidade e valor preditivo desses exames. Os profissionais de saúde ocupacional não devem utilizar testes de rastreamento nem realizar investigações que não sejam confiáveis ou que não tenham um valor preditivo suficiente em relação aos requisitos e atribuições de uma atividade de trabalho ${ }^{47}$.

O modelo de decisão que está se propondo aplica-se para avaliações médicas no exame admissional. Se o trabalhador e sua condição clínica já são de conhecimento do médico do trabalho ou se a patologia que pode culminar em evento agudo e levar à queda do trabalhador já foi diagnosticada no exame admissional, não é necessário repetir o exame diagnóstico nas avaliações periódicas futuras.

Para a aplicação do modelo em exames periódicos, seria necessário outro estudo, levando em consideração outras variáveis. Nesse caso, também se deve considerar a classificação do grau de risco obtida pela aplicação dos critérios do princípio ALARP para determinado evento de saúde (inaceitável, tolerável, amplamente aceitável), bem como a patologia e o teste diagnóstico. Porém, trata-se de outro cenário, pois a probabilidade considerada para a ocorrência de um evento agudo de saúde será diferente no admissional e no periódico. No exame admissional, utiliza-se a prevalência do evento estudado na população como um todo, uma vez que se trata de uma população desconhecida que chega ao médico e que pode ou não apresentar determinada patologia. No exame periódico, admitindo-se que o trabalhador não possuía a doença estudada no momento da contratação, deve ser considerada a probabilidade de um indivíduo sem a doença passar a ser acometido por ela.

\section{Aplicação do modelo proposto para casos de diabetes mellitus}

\section{Etapa 1: Caracterização do evento agudo de saúde}

A hipoglicemia é o efeito adverso mais comum no tratamento de diabetes com insulina e sulfonilureias. Alterações de equilíbrio, coordenação, visão e nível de consciência decorrentes da hipoglicemia podem precipitar quedas e ferimentos ${ }^{48-50}$. 
A prevalência de DM apresenta diferenças marcantes entre diversos países e grupos étnicos. Adotou-se, neste estudo, a prevalência de DM1 de 0,2\% e frequência de 3,2 episódios de hipoglicemia por paciente por ano. Para DM2, prevalência de $8,35 \%$, sendo que, dos pacientes acometidos de DM2, 13,5\% faziam uso de insulinoterapia e apresentavam 0,7 episódios de hipoglicemia por paciente por ano, e 13\% utilizavam sulfonilureia, com 0,1 episódios de hipoglicemia por paciente por ano ${ }^{12-14,16,17}$.

Etapa 2: Caracterização do evento agudo de saúde em termos de riscos e tomada de decisão

A probabilidade de ocorrência de um evento hipoglicêmico entre os candidatos ao trabalho em altura decorrente de DM1 é obtida pelo produto da prevalência de DM1 pela frequência de episódios de hipoglicemia por ano em diabéticos tipo 1, como demonstrado no cálculo apresentado na Equação 1 (Quadro 1).

Assim, de cada 1.000 indivíduos da população em geral a serem admitidos no trabalho, a probabilidade de ocorrência de hipoglicemia seria de 6,4 episódios por ano, ou seja, 6,4 episódios por 1.000 trabalhadores por ano. Como a ocorrência de um evento agudo de saúde (episódio de hipoglicemia) durante o trabalho em altura será considerada equivalente a um acidente fatal, isto representaria 6,4 acidentes fatais por 1.000 indivíduos por ano.

Para determinar a tolerabilidade do risco desse evento, deve-se comparar a probabilidade encontrada com os critérios de tolerabilidade do princípio ALARP. Neste caso, observa-se que o risco de evento hipoglicêmico em decorrência de DM1 pertence à faixa superior do triângulo de representação, ou seja, acima do limite de risco máximo tolerável, sendo caracterizado como risco inaceitável (Figura 3).

Já em relação ao DM2 em tratamento com insulina, a probabilidade de ocorrência de um evento hipoglicêmico entre trabalhadores a serem admitidos para o trabalho será obtida como mostrado a seguir:

Probabilidade de ocorrência de um evento hipoglicêmico decorrente de DM2 em insulinoterapia = prevalência de DM2 $\times$ prevalência de diabéticos tipo 2 em insulinoterapia $\times$ frequência de episódios de hipoglicemia/ano em diabéticos tipo 2 em insulinoterapia. O cálculo é apresentado na Equação 2 (Quadro 1).

Ou seja, a probabilidade de ocorrência de um evento hipoglicêmico decorrente de DM2 em insulinoterapia é de 7,9 episódios de hipoglicemia por 1.000 indivíduos por ano, o que seria equivalente a 7,9 acidentes fatais por 1.000 trabalhadores por ano.

Para DM2 em tratamento com sulfonilureia, a probabilidade de ocorrência de um evento hipoglicêmico em decorrência dessa condição clínica entre trabalhadores a serem admitidos para o trabalho é calculada pela probabilidade de ocorrência de um evento hipoglicêmico decorrente de DM2, em monoterapia com sulfonilureia, sendo resultado da prevalência de DM2 $\times$ prevalência de diabéticos tipo 2 em monoterapia com sulfonilureia $\times$ frequência de episódios de hipoglicemia/ano em diabéticos tipo 2 em monoterapia com sulfonilureia. O cálculo é apresentado na Equação 3 (Quadro 1).

Quadro 1 Equações de cálculo de probabilidade de ocorrência de hipoglicemia decorrente de diabetes mellitus

\begin{tabular}{|c|c|}
\hline Equação & Cálculo \\
\hline $\begin{array}{l}1 \\
(\mathrm{DM} 1 *)\end{array}$ & $\begin{array}{c}\frac{0,2 \text { indiv. DM1 }}{100 \text { indiv. da pop. geral }} \times \frac{3,2 \text { episódios hipoglicemia }}{1 \text { indiv. DM1 } / \text { ano }}=\frac{0,0064 \text { episódios }}{\text { indiv. } / \text { ano }} \\
=6,4 \text { episódios } / 1.000 \text { indiv. } / \text { ano }\end{array}$ \\
\hline$\left(\right.$ DM2- insulina $\left.{ }^{\alpha}\right)$ & $\begin{array}{c}\frac{8,35 \text { indiv. DM2 }}{100 \text { indiv. da pop. geral }} \times \frac{13,5 \text { indiv. DM2 em uso de insulina }}{100 \text { indiv. DM2 }} \times \frac{0,7 \text { episódios }}{1 \text { indiv. DM2 em uso de insulina } / \text { ano }} \\
=0,0079 \text { episódios } / \text { indiv. } / \text { ano }=7,9 \text { episódios } / 1.000 \text { indiv. } / \text { ano }\end{array}$ \\
\hline$\left(\right.$ DM2- sulfonilureia $\left.{ }^{\beta}\right)$ & $\begin{array}{c}\frac{8,35 \text { indiv. DM2 }}{100 \text { indiv. da pop. geral }} \times \frac{13 \text { indiv. DM2 em uso de sulfonilureia }}{100 \text { indiv. DM2 }} \times \frac{0,1 \text { episódios }}{1 \text { indiv. DM2 em uso de sulfonilureia/ano }} \\
=0,00108 \text { episódios } / \text { indiv. } / \text { ano }=1,08 \text { episódios } / 1.000 \text { indiv. } / \text { ano }\end{array}$ \\
\hline
\end{tabular}


Ou seja, para DM2 em tratamento com sulfonilureia, a probabilidade de ocorrência de um evento hipoglicêmico será de 1,08 episódios de hipoglicemia por 1.000 indivíduos por ano, ou o que seria equivalente 1,08 acidentes fatais por 1.000 trabalhadores por ano.

Comparando esses valores com os critérios de tolerabilidade do princípio ALARP, observa-se que os riscos de eventos hipoglicêmicos em decorrência de DM2, tanto em tratamento com insulina como com sulfonilureia, são numericamente maiores que 1 acidente fatal por 1.000 trabalhadores por ano, estando acima do nível de risco individual máximo tolerável para trabalhadores e também pertencendo à faixa de risco inaceitável (Figura 3).

Conforme o princípio ALARP, sendo o risco considerado inaceitável, o seu tratamento é indispensável. Nesses casos, a atividade de trabalho só pode ser executada se as medidas de controle reduzirem o grau de risco para níveis inferiores.

Desta forma, observa-se que para um paciente acometido de DM1 ou DM2 executar trabalho em altura, deverá ser feita uma abordagem de modo a controlar o risco de ocorrência de acidentes provocados por eventuais eventos hipoglicêmicos visando diminuir o risco para um valor abaixo do valor máximo tolerável do princípio ALARP. Essa abordagem se dá por medidas de segurança, que são imprescindíveis, e, em relação à saúde ocupacional, pelo diagnóstico de diabetes durante a avaliação médica admissional. Naturalmente, para identificar quem tem risco de sofrer hipoglicemia, deve-se diagnosticar o DM e caracterizá-lo previamente.

Etapa 3: Avaliar as opções disponíveis para o gerenciamento de riscos no âmbito dos exames médicos ocupacionais

Uma vez identificado como um risco inaceitável, o evento hipoglicêmico em diabéticos deve ser abordado a fim de diminuir o risco de acidentes fatais. Antes da efetivação da abordagem, é preciso avaliar de que maneira deve ser realizada.

A Sociedade Brasileira de Diabetes ${ }^{23}$ e a American Diabetes Association ${ }^{19}$, em suas diretrizes, colocam que o método preferencial para determinação da glicemia é sua aferição no plasma.

A aferição da glicose no plasma de indivíduos em jejum - glicemia de jejum (GJ) - é amplamente aceita como critério diagnóstico de diabetes. Entre as vantagens na utilização desse teste estão o baixo custo, a necessidade de apenas uma amostra de sangue e a fácil disponibilidade, além de que os estudos de ensaios serem facilmente realizados com instrumentos automatizados que estão disponíveis na maioria dos laboratórios de todo o mundo $21,22,25$. No entanto, a GJ está sujeita a algumas limitações, e seu resultado pode ser influenciado por variações biológicas, pré-analíticas e analíticas ${ }^{22}$.

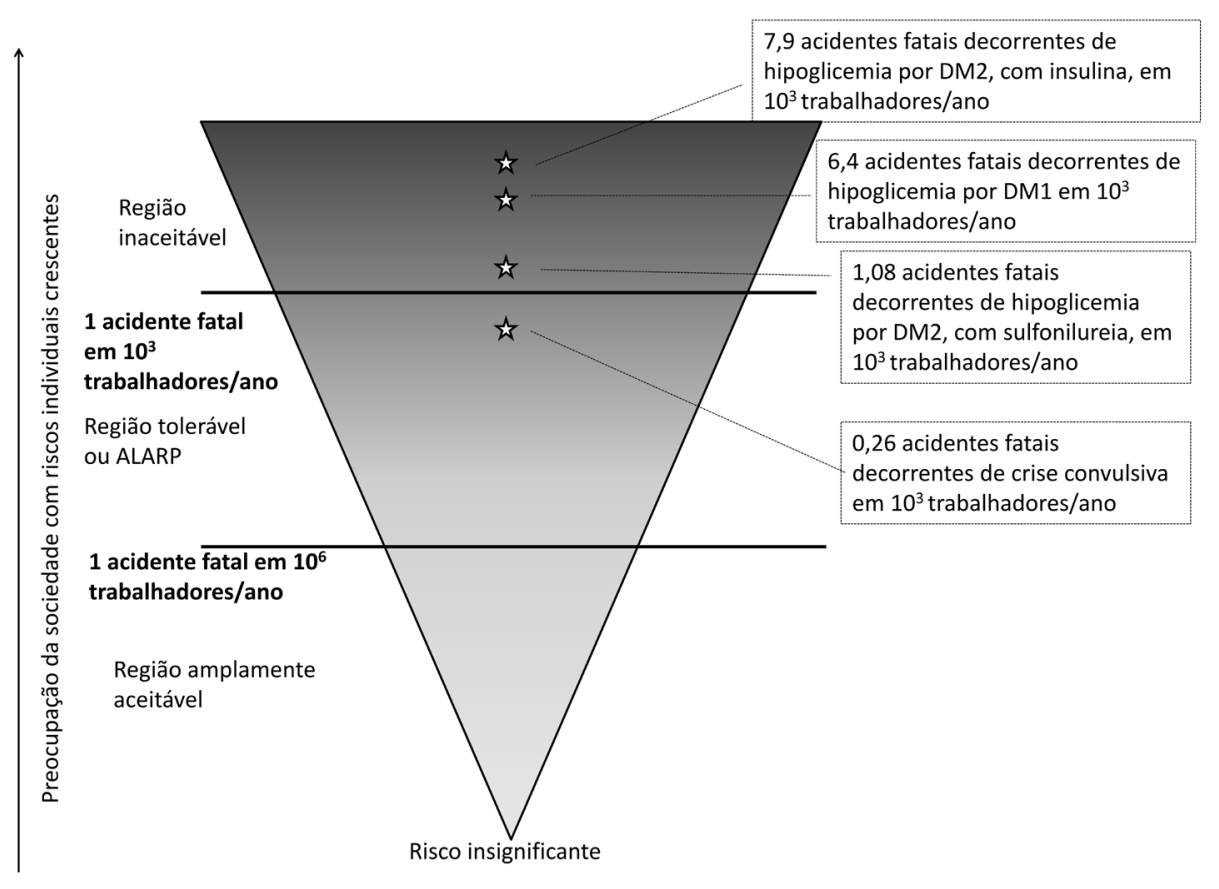

Figura 3 Identificação da magnitude do risco de evento hipoglicêmico decorrente de DM1 e DM2* e do risco de evento de crise convulsiva decorrente de epilepsia no modelo esquemático do princípio ALARP ${ }^{* *}$

Notas: " DM1 e DM2: Diabetes mellitus tipos 1 e 2

****ALARP: As Low As Reasonably Practicable, critério de identificação de tolerabilidade de risco ${ }^{11}$ 
A dosagem de GJ apresenta uma sensibilidade entre $40 \%$ e $65 \%$, com uma especificidade maior que $90 \%$ para valores variando de 108 a $126 \mathrm{mg} / \mathrm{dL}^{24}$. Blunt et al. ${ }^{20}$ relatam especificidade de uma única dosagem de GJ com um ponto de corte de $126 \mathrm{mg} /$ $\mathrm{dL}$ como sendo maior que $95 \%$ e a sensibilidade de cerca de $50 \%$, quando comparado com o teste oral de tolerância à glicose (TOTG).

No Brasil, a Sociedade Brasileira de Diabetes ${ }^{23}$ considera que são necessários mais estudos para a utilização da hemoglobina glicada (HbA1c) como critério de diagnóstico para o DM.

Selph et al. ${ }^{37}$ relatam que existem evidências limitadas sobre os danos da triagem para o DM. Os autores não identificaram nenhum estudo relatando efeitos adversos psicológicos sérios ou outros efeitos associados com a triagem ou um novo diagnóstico de DM. Referem ter encontrado três estudos que abordam os efeitos psicológicos leves associados à triagem ou a um novo diagnóstico de DM, sendo que em um deles houve relação entre ansiedade de curto prazo e o convite para o rastreamento e um novo diagnóstico da doença, e em dois estudos de longa duração não foram encontrados efeitos psicológicos negativos associados. Também não foram identificados estudos sobre danos associados a testes falso-positivos para DM.
Dessa maneira, a GJ é o teste mais indicado para o diagnóstico de DM em uma avaliação ocupacional.

Uma vez que o DM seja diagnosticado no exame admissional de indivíduos que executam atividades críticas, o trabalhador deve ser encaminhado a um especialista para tratamento e acompanhamento. Caso seja prescrito uso de insulina ou sulfonilureia, é importante que o fato seja de conhecimento do médico do trabalho, que, então, deverá questionar e averiguar o paciente por meio da anamnese ocupacional.

Não é necessária a repetição do teste diagnóstico nas avaliações periódicas subsequentes caso se tenha diagnosticado DM no exame admissional. Em relação à utilização desse teste no exame periódico para aqueles não diagnosticados com DM, é necessário outro estudo, levando em consideração a probabilidade de um indivíduo sem a doença desenvolver DM dentro do intervalo de tempo entre o admissional e o periódico. O trabalhador diabético que tenha sido considerado apto no exame admissional por não utilizar insulina ou sulfonilureia também deve ter atenção especial no exame periódico para se certificar de que não adquiriu a condição.

Um desenho esquemático do modelo proposto aplicado ao evento hipoglicêmico decorrente de diabetes é apresentado na Figura 4.

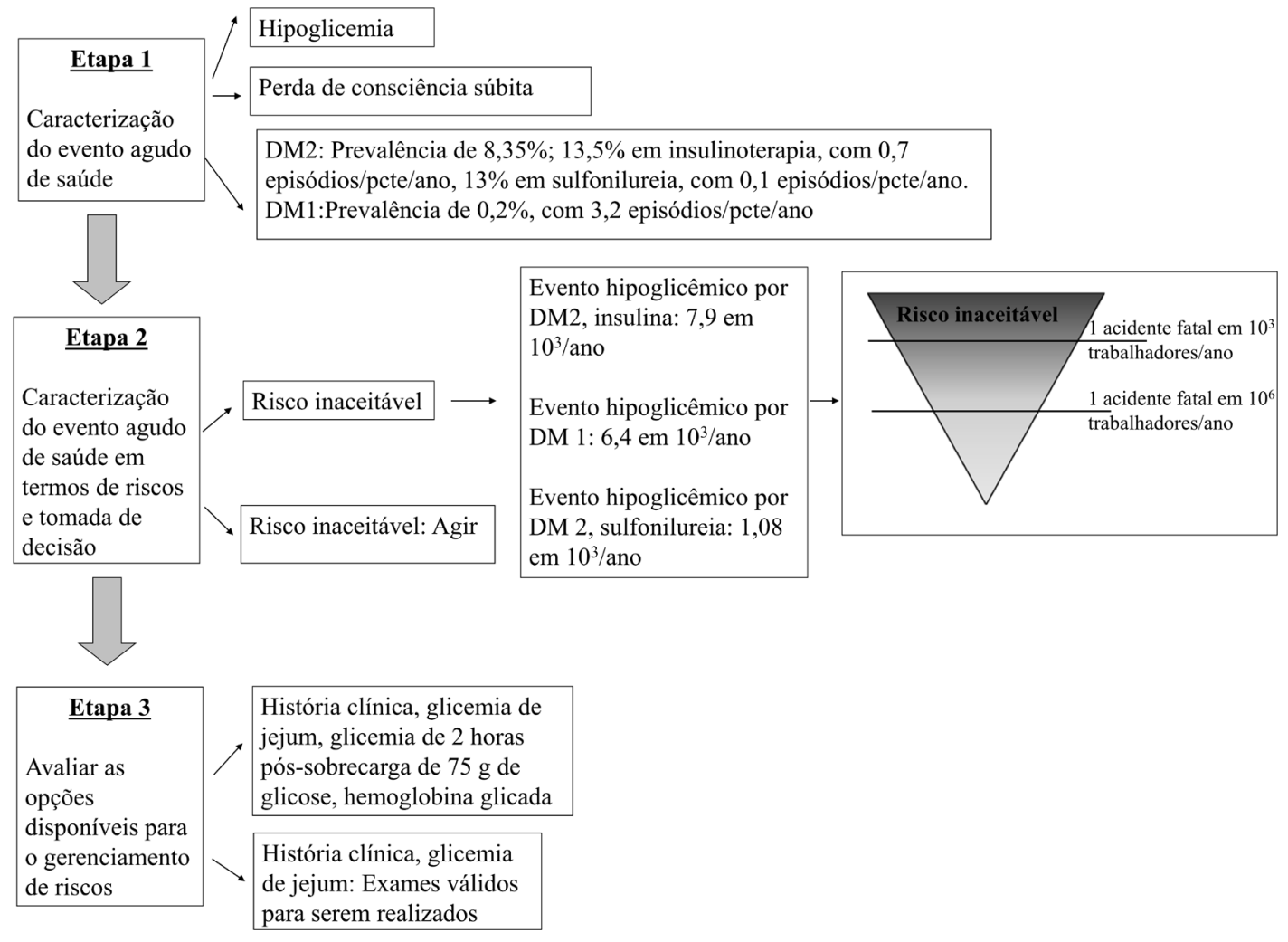

Figura 4 Modelo de tomada de decisão aplicado ao evento hipoglicêmico decorrente de diabetes mellitus tipo 1 (DM1) e tipo 2 (DM2), para a definição de exames médicos ocupacionais de avaliação de trabalhadores que executam atividades críticas 


\section{Aplicação do modelo proposto na epilepsia}

\section{Etapa 1: Caracterização do evento agudo de saúde}

A incidência, prevalência e mortalidade da epilepsia variam entre os países com economias diferentes ${ }^{51}$. Adotou-se, neste estudo, a prevalência de epilepsia de 16,5/1.000 pessoas e frequência de 0,26 episódios de crise convulsiva por paciente por ano ${ }^{15,18}$

Os acidentes e lesões são ligeiramente mais frequentes em pessoas com epilepsia do que na população em geral, provavelmente com maior risco em epilépticos sintomáticos que apresentam crises epilépticas frequentes e quando as crises estão associadas a deficiências. Porém, em relação a acidentes de trabalho, quando se excluem as pessoas com epilepsia refratária ou com doenças neurológicas, psiquiátricas e deficiências associadas, a epilepsia, parece não haver risco aumentado de acidentes no trabalho ${ }^{52}$.

Etapa 2: Caracterização do evento agudo de saúde em termos de riscos e tomada de decisão

A probabilidade de ocorrência de uma crise convulsiva em decorrência de epilepsia entre trabalhadores a serem admitidos para o trabalho é calculada abaixo:

Probabilidade de ocorrência de uma crise convulsiva decorrente de epilepsia = prevalência de epilepsia $\times$ frequência de episódios de crise convulsiva/ paciente/ano.

Então, a probabilidade de ocorrência de uma crise convulsiva é de 0,26 episódios de crise convulsiva por 1.000 indivíduos por ano, que pode ser considerada equivalente a 0,26 acidentes fatais por 1.000 trabalhadores por ano. O cálculo é apresentado na Equação 4 (Quadro 2).

Comparando esse valor com os critérios de risco do princípio ALARP, observa-se que o risco de evento de crise convulsiva em decorrência de epilepsia está na faixa intermediária, ou região tolerável (Figura 3).

Conforme já discutido, na região tolerável a magnitude dos riscos é menos elevada do que na região denominada inaceitável, e deve ser mantida tão baixa quanto razoavelmente praticável, devendo-se ponderar a redução em relação ao custo-benefício para tal redução.
Etapa 3: Avaliar as opções disponíveis para o gerenciamento de riscos no âmbito dos exames médicos ocupacionais

O diagnóstico de epilepsia é clínico e realizado por anamnese e exame físico detalhados. Se necessários, os exames complementares devem ser orientados pelos achados da história e do exame físico $26-29,31$.

O eletroencefalograma (EEG) interictal é relativamente barato e de fácil obtenção em esquema ambulatorial, sem necessidade de internação para realização. Porém, o exame está sujeito a algumas limitações que podem alterar seu resultado, uma vez que representa um registro pontual, podendo não detectar descargas epileptiformes interictais que ocorrem com pouca frequência. Ainda, os eletrodos no couro cabeludo cobrem apenas um terço do córtex cerebral, portanto as descargas que ocorrem dentro de sulcos, regiões basais ou inter-hemisféricas podem não ser detectadas. Além disso, a calota craniana, dura-máter e tecido do couro cabeludo podem atenuar os sinais e o resultado é observador-dependente, podendo ocorrer erros de interpretação do EEG ${ }^{31}$.

Segundo o National Clinical Guideline Centre ${ }^{28}$, a sensibilidade do exame varia de $26 \%$ a $56 \%$, a especificidade de $78 \%$ a $98 \%$, a razão de verossimilhança positiva varia de 2,5 a 13 e a razão de verossimilhança negativa, de 0,5 a 0,76 . O achado de atividade epileptiforme interictal no EEG pode ser usado para ajudar a confirmar o diagnóstico clínico de crise epiléptica. Porém, um EEG negativo não pode ser utilizado para descartar o diagnóstico clínico de epilepsia. O estudo ainda reforça que o EEG não deve ser utilizado isoladamente para fazer o diagnóstico de epilepsia.

Em uma revisão sobre a prevalência de descargas epileptiformes em indivíduos sem antecedente de crise convulsiva realizada por $\mathrm{So}^{35}$, foram encontrados estudos que mostram prevalência de descargas espontâneas em adultos voluntários saudáveis variando de $0 \%$ a $6,6 \%$. Entre candidatos a piloto de aviação, que são submetidos ao EEG para triagem, a taxa encontrada foi de $0,5 \%$ a $2,5 \%$. O autor refere que nesta população existem poucos estudos sobre a ocorrência de crises convulsivas após a detecção de descargas epileptiformes no EEG, pois o follow-up raramente é realizado para esses candidatos que, por apresentarem descargas no EEG de triagem, são considerados inaptos. Um dos estudos analisados pelo autor aponta que 1 em cada 20 candidatos com EEG alterado apresentou crise convulsiva nos 10 anos que se seguiram ao exame, enquanto outro aponta que nenhum indivíduo com alteração no exame apresentou crise convulsiva nos 10 ou 15 anos seguintes.

Quadro 2 Equação de cálculo de probabilidade de ocorrência de crise convulsiva decorrente de epilepsia

\begin{tabular}{|cc|}
\hline Equação & Cálculo \\
\hline 4 & $\frac{16,5 \text { pacientes com epilepsia }}{1.000 \text { indiv. da pop. geral }} \times \frac{0,016 \text { episódios }}{\text { paciente com epilepsia/ano }}=0,26$ episódios $/ 1.000$ indiv. $/$ ano \\
(epilepsia) &
\end{tabular}


É importante discernir se trata-se de um paciente com suspeita, queixa ou história prévia de alterações neurológicas ou de um indivíduo sem essas condições. Essas informações são obtidas por anamnese e exame físico. Enquadram-se como pacientes todos os com história de crises epilépticas, de epilepsia, de manipulação neurocirúrgica, com comprometimento do segmento crânio-encefálico (metabólico, infeccioso, tumoral, vascular) e internados com alteração comportamental ou rebaixamento do nível de consciência. Quando utilizado para esses pacientes, o EEG pode apresentar especificidade de até 98\% em uma relação exponencial, ou seja, quanto maior for a suspeita clínica, maior será a proporção de verdadeiros positivos. Assim, nesses casos, o EEG seria indicado $^{32,33}$. Entretanto, não há evidências claras de que a triagem com EEG em indivíduos saudáveis possa levar à redução significativa do risco de acidentes, uma vez que, utilizado desta maneira, o exame apresenta baixo valor preditivo. Dessa maneira, não há indicação de EEG para essa população ${ }^{28,30,32-34,36}$.

Assim, fica evidente que a solicitação do EEG para todos os candidatos antes do exame médico admissional não é indicada, sendo de extrema importância a obtenção da história clínica durante a anamnese ocupacional. É necessário cautela durante uma investigação com EEG quando a história clínica é negativa para epilepsia, uma vez que o risco de um resultado falso-positivo pode levar a erros de diagnóstico. Um diagnóstico errado pode tornar um indivíduo que não tem doença em doente e, muitas vezes, leva-lo à exclusão do mercado de trabalho.

Em relação à utilização desse teste, deve-se considerar que a quantidade de doentes atendidos pelo médico do trabalho no exame periódico e no admissional difere. Neste, a população que chega é desconhecida, podendo ou não apresentar epilepsia. No periódico, serão examinados os avaliados no admissional e considerados sem doença, ou uma pequena parcela que desenvolveu a doença dentro do intervalo de tempo entre o admissional e o periódico. Além disso, o mesmo raciocínio em relação às características e validade do EEG se aplica ao exame admissional e periódico. Dessa maneira, tanto no admissional como no periódico, o importante é obter informações clínicas a partir da anamnese e exame físico, para que o médico saiba se está diante de um indivíduo saudável ou de um paciente, o que altera a indicação de solicitação do EEG.

Na Figura 5 apresenta-se resumidamente um desenho esquemático do modelo proposto aplicado ao evento de crise convulsiva decorrente de epilepsia.

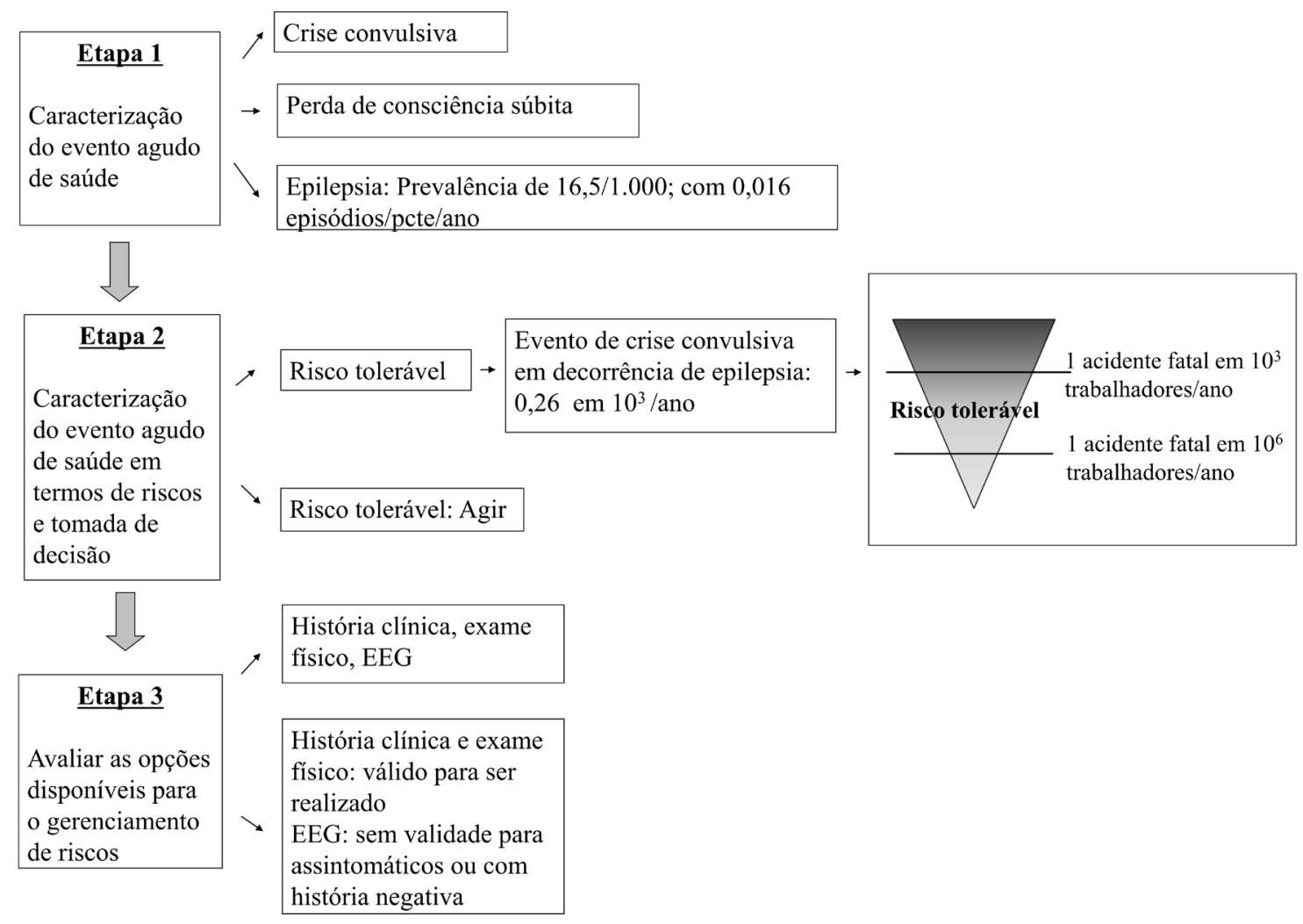

Figura 5 Modelo de tomada de decisão aplicado ao evento de crise convulsiva decorrente de epilepsia para a definição de exames médicos ocupacionais de avaliação de trabalhadores que executam atividades críticas 


\section{Considerações finais}

É preciso saber buscar as melhores evidências e aplicá-las, além de ser essencial dispensar tempo suficiente ao trabalhador durante a avaliação ocupacional, com seu devido acolhimento, escuta atenta, anamnese e exame físico completo. Deve-se também prestar atenção ao registro adequado no prontuário médico. Também é necessário conhecer de fato a atividade a ser realizada, as condições e o ambiente de trabalho.

Independentemente do fator de saúde que possa levar à instabilidade postural durante uma atividade de trabalho em altura, é essencial que sejam cumpridos os requisitos mínimos da NR35 e as medidas de proteção para o trabalho em altura. Para o cumprimento dos requisitos e das medidas de proteção, é imprescindível considerar o planejamento, a organização e a execução da atividade, de forma que sejam asseguradas a segurança e a saúde dos trabalhadores direta ou indiretamente envolvidos.

Os objetivos do presente ensaio foram alcançados. A partir do referencial preconizado pela HSE, foi proposto um modelo de decisão para a definição de exames médicos ocupacionais para avaliar a aptidão de trabalhadores que exercem atividades críticas e foi verificada a sua aplicação para o caso do trabalho em altura com exemplificação em duas condições clínicas potencialmente desencadeantes de acidentes fatais. Observou-se ser possível embasar a avaliação médica ocupacional por meio da utilização de parâmetros e de evidências científicas para a tomada de decisão.
Verificou-se tratar-se de um modelo que pode subsidiar a decisão do médico do trabalho em relação à necessidade de investigar ou não determinadas condições clínicas que possam levar a eventos agudos, no caso, aqueles que podem afetar a estabilidade postural do trabalhador durante a realização de atividades em altura, podendo gerar acidente fatal.

Os resultantes da aplicação do modelo proposto, sobre como interpretar as informações disponíveis relacionadas aos eventos de saúde potencialmente causadores de acidentes e sobre definir a necessidade de fazer ou não determinados exames médicos, devem ser revistos periodicamente, uma vez que novos dados e novas tecnologias em saúde vão surgindo com o avanço da ciência médica, além da constante mudança no perfil demográfico e epidemiológico da população.

Os critérios de níveis de tolerabilidade de riscos devem ser coerentes com os valores da sociedade. Com o tempo, ocorrem mudanças de padrões e das expectativas do público, e o que antes era inaceitável pode passar a ser tolerável, e o que é tolerável pode deixar de ser. Assim, cabe à sociedade discutir os níveis de tolerabilidade e definir o quanto está disposta a conviver com um determinado risco.

Entende-se que o modelo de decisão proposto seja apenas um ponto de partida para outros estudos, como a sua aplicação sob outras condições clínicas que podem levar à queda do trabalhador e também com a aplicação em outras atividades críticas de trabalho. Espera-se que o presente ensaio possa trazer contribuições para o debate e estimular estratégias acerca da prevenção de acidentes e promoção da saúde e a segurança dos trabalhadores.

\section{Contribuições de autoria}

Hayashide JM participou da concepção metodológica do projeto, do levantamento e análise dos dados, da redação e da revisão e aprovação final do artigo. Buschinelli JTP participou da concepção metodológica do projeto, da análise dos dados, da revisão e aprovação final do artigo.

\section{Referências}

1. Alcoa. Guia de requisitos mínimos obrigatórios de SSMA - segurança, saúde e meio ambiente para contratadas. Poços de Caldas: Alcoa; 2001 [citado em 15 abr 2014]. Disponível em: http://bit. ly/2jzYJlV

2. Vale. Instrução para requisitos de atividades críticas. Rio de Janeiro: Vale; 2009 [citado em 7 abr 2014]. Disponível em: http://bit.ly/2kUWJ82

3. Brasil. Portaria da Secretaria de Inspeção do Trabalho n. ${ }^{\circ}$ 313, de 23 de março de 2012. Aprova a Norma Regulamentadora n. ${ }^{\circ} 35$ (trabalho em altura). Diário Oficial [da] República Federativa do Brasil, Poder Executivo, Brasília, DF; 27 mar 2012. Seção 1, p. 140.

4. Health and Safety Executive. United Kingdom Legislation. The work at height regulations 2005. United Kingdom; 2005 [citado em 7 dez 2013]. Disponível em: http://bit.ly/2k3hkpd

5. Occupational Safety \& Health Administration. Safety and health regulations for construction. 
Washington, DC; 1995 [citado em 5 dez 2013]. Disponível em: http://bit.ly/1zQfQAI

6. Canadian Centre of Occupational Health and Safety. Body belts, harnesses, and lanyards. 2013 [citado em 8 jun 2014]. Disponível em: http://www. ccohs.ca/oshanswers/prevention/ppe/belts.html

7. Health and Safety Executive. United Kingdom Legislation. Kinds of accident in Great Britain, 2013. United Kingdom; 2013 [citado em 5 dez 2013]. Disponível em: http://bit.ly/1MBAiXt

8. United States Department of Labor. National census of fatal occupational injuries in 2013: preliminary results. Bureau of Labor Statistics, 2014 [citado em 21 jun 2015]. Disponível em: http://bit.ly/2jGWtEF

9. Brasil. Ministério do Trabalho e Emprego. NR-35 Trabalho em altura comentada. Brasília, DF; 2013 [citado em 5 dez 2013]. Disponível em: http://bit. ly/2kZLuHo

10. Health and Safety Executive. United Kingdom Legislation. Falls from height: prevention and risk control effectiveness. United Kingdom; 2003 [citado em 8 dez 2013]. Disponível em: http://bit. ly/2jZlwUh

11. Health and Safety Executive. United Kingdom Legislation. Reducing risks, protecting people: HSE's decision-making process. United Kingdom; 2001 [citado em 12 abr 2014]. Disponível em: http://bit.ly/2jH6PEk

12. International Diabetes Federation. Diabetes atlas. 2013 [acesso em 14 jun 2014]. Disponível em: http://bit.ly/1iiBkyH

13. Virally M, Blicklé JF, Girard J, Halimi S, Simon D, Guillausseau PJ. Type 2 diabetes mellitus: epidemiology, pathophysiology, unmet needs and therapeutical perspectives. Diabetes Metab. 2007;33(4):231-44

14. Brouwer ES, West SL, Kluckman M, Wallace D, Masica AL, Ewen E, Kudyakov R, Cheng D, Bowen J, Fleming NS. Initial and subsequent therapy for newly diagnosed type 2 diabetes patients treated in primary care using data from a vendor-based electronic health record. Pharmacoepidemiol Drug Saf. 2012;21(9):920-8

15. Fernandes J, Schmidt M, Monte T, Tozzi S, Sander J. Prevalence of epilepsy: the Porto Alegre study. Epilepsia. 1992;33(Suppl 3):132.

16. UK Hypoglycaemia Study Group. Risk of hypoglycaemia in types 1 and 2 diabetes: effects of treatment modalities and their duration. Diabetologia. 2007;50(6):1140-7.

17. Schopman JE, Simon AC, Hoefnagel SJ, Hoekstra JB, Scholten RJ, Holleman F. The incidence of mild and severe hypoglycaemia in patients with type 2 diabetes mellitus treated with sulfonylureas: a systematic review and meta-analysis. Diabetes Metab Res Rev. 2014;30(1):11-22.

18. Takeda A, Kawai I, Fukushima Y, Yagi K. Driving and epilepsy: a prospective questionnaire survey in Japan. Jpn J Psychiatry Neurol. 1991;45(2):319-22.
19. American Diabetes Association. Standards of medical care in diabetes: 2014. Diabetes Care. 2014;37(Suppl. 1):S14-S80.

20. Blunt BA, Barrett-Connor E, Wingard DL. Evaluation of fasting plasma glucose as screening test for NIDDM in older adults: Rancho Bernardo study. Diabetes Care. 1991;14(11):989-93.

21. Norris SL, Kansagara D, Bougatsos C, Nygren P, Fu R. Screening for type 2 Diabetes Mellitus: update of 2003 systematic evidence review for the U.S. Preventive Services Task Force. AHRQ Publication No. 08-05116-EF-1. Rockville: Agency for Healthcare Research and Quality; 2008.

22. Sacks DB. A1C versus glucose testing: a comparison. Diabetes Care. 2011;34(2):518-23.

23. Sociedade Brasileira de Diabetes. Diretrizes da Sociedade Brasileira de Diabetes: 2013-2014. São Paulo: AC Farmacêutica; 2014.

24. World Health Organization. Screening for Type 2 Diabetes: report of a World Health Organization and International Diabetes Federation meeting. 2003. Geneva: World Health Organization; 2003 [acesso em 7 set 2015]. Disponível em: http://bit. $\mathrm{ly} / 2 \mathrm{k} 01 \mathrm{HfB}$

25. World Health Organization. Use of glycated haemoglobin (HbA1c) in the diagnosis of Diabetes Mellitus: abbreviated report of a WHO consultation. Geneva: World Health Organization, 2011 [acesso 7 set 2015]. Disponível em: http://bit. ly/297SQTq

26. Brasil. Ministério da Saúde. Secretaria de Atenção à Saúde. Portaria $\mathrm{n}^{0} 1.319$, de 25 de novembro de 2013. Aprova o Protocolo Clínico e Diretrizes Terapêuticas da Epilepsia. Diário Oficial [da] República Federativa do Brasil, Poder Executivo, Brasília, DF; 27 nov. 2013. Seção 1, p. 138-143.

27. Fisher RS, Acevedo C, Arzimanoglou A, Bogacz A, Cross JH, Elger CE, Engel Jr J, Forsgren L, French JA, Glynn M, Hesdorffer DC, Lee BI, Mathern GW, Moshé SL, Perucca E, Scheffer IE, Tomson T, Watanabe M, Wiebe S. A practical clinical definition of epilepsy. Epilepsia. 2014;55(4):47582.

28. National Clinical Guideline Centre. The Epilepsies: the diagnosis and management of the epilepsies in adults and children in primary and secondary care. London; 2012 [citado em 15 jun 2014]. Disponível em: http://bit.ly/2k4my41

29. Noachtar S, Rémi J. The role of EEG in epilepsy: a critical review. Epilepsy Behav. 2009;15(1):22-33.

30. Nunes S, De Oliveira SN, Rosado P. Electroencefalograma interictal: sensibilidade e especificidade no diagnóstico de epilepsia. Acta Med Port. 2004;17:465-70.

31. Pillai J, Sperling MR. Interictal EEG and the diagnosis of epilepsy. Epilepsia. 2006;47(Suppl 1):14-22.

32. Reunião científica sobre "Trabalho em altura: o que seria razoável no exame médico ocupacional do ponto de vista neurológico. Deve ser feito o EEG?”, 
2014, São Paulo. Material discutido... São Paulo: Associação Paulista de Medicina do Trabalho, 2014. Disponível em: <http://bit.ly/2kklt9X>. Acesso em: 15 abr. 2014.

33. Reunião científica sobre "trabalho em altura: o que seria razoável no exame médico ocupacional do ponto de vista neurológico. Deve ser feito o EEG?”, 2014b, São Paulo. Relato da reunião... São Paulo: Associação Paulista de Medicina do Trabalho, 2014. Disponível em: < http://bit.ly/2kkmPS9>. Acesso em: 15 abr. 2014.

34. São Paulo. Conselho Regional de Medicina. Consulta no 40.007/96. São Paulo: Cremesp; 1996 [citado em 15 fev 2015]. Disponível em: http://bit.ly/2loGIcG

35. So EL. Interictal epileptiform discharges in persons without a history of seizures: what do they mean? J Clin Neurophysiol. 2010;27(4):229-38.

36. Zifkin BG. The electroencephalogram as a screening tool in pilot applicants. Epilepsy Behav. 2005;6(1):17-20.

37. Selph S, Dana T, Bougatsos C, Blazina I, Patel H, Chou R. Screening for abnormal glucose and type 2 Diabetes Mellitus: a systematic review to update the 2008 U.S. Preventive Services Task Force recommendation. Evidence Synthesis No. 117. AHRQ Publication No. 13-05190- EF-1. Rockville: Agency for Healthcare Research and Quality; 2015.

38. International Electrotechnical Comission. International Organization for standardization. IEC/ISO 31010: risk management — risk assessment techniques. Switzerland: ISO; 2009.

39. Rosa AC. Risco tecnológico em contextos de vulnerabilidade social e ambiental elevados: experiências na região da refinaria Gabriel Passos/ Petrobras, região metropolitana de Belo Horizonte, Minas Gerias (1998-2007) [dissertação]. Ouro Preto: Universidade Federal de Ouro Preto; 2008 [citado em 12 jun 2014]. Disponível em: http://bit. ly/2kWuSUW

40. International Organization for Standardization. ISO Guide 73: risk management: vocabulary. Switzerland: ISO; 2009.
41. Center for Chemical Process Safety. Guidelines for developing quantitative safety risk criteria. New Jersey: John Wiley \& Sons; 2009.

42. Health and Safety Executive. The tolerability of risk from nuclear power stations. United Kingdom: HSE; 1992 [citado em 20 abr 2016]. Disponível em: http://bit.ly/2kWowVU

43. Fletcher RH, Fletcher SW, Fletcher GS Epidemiologia clínica: elementos essenciais. 5. ed. Porto Alegre: Artmed, 2014.

44. Atallah AN, Castro AA. Medicina baseada em evidências: o elo entre a boa ciência e a boa prática. Revista da Imagem. 1998;20(1):V-IX.

45. Alves R. Estudos transversais de prevalência e de diagnóstico. Residência Pediátrica. 2012;2(1):9-11.

46. Fiszman R, Matos MFD, De Souza e Silva NA. Análise crítica do uso de exames complementares na prática médica. Rev Bras Cardiol. 2003;16(2):101-9.

47. International Commission on Occupational Health. International code of ethics for occupational health professionals. Roma: Icoh; 2014 [citado em 20 abr 2015]. Disponível em: http://bit.ly/2l3DfLp

48. Frier BM. Hypoglycaemia in diabetes mellitus: epidemiology and clinical implications. Nat Rev Endocrinol. 2014;10(12):711-22.

49. Graveling AJ, Frier BM. Hypoglycaemia: an overview. Prim Care Diabetes. 2009;3(3):131-9.

50. Seaquist ER, Anderson J, Childs B, Cryer P, Dagogo-Jack S, Fish L, Heller SR, Rodriguez H, Rosenzweig J, Vigersky R. Hypoglycemia and diabetes: a report of a workgroup of the American Diabetes Association and the Endocrine Society. Diabetes Care. 2013;36(5):1384-95.

51. Beghi E, Hesdorffer D. Prevalence of epilepsy: an unknown quantity. Epilepsia. 2014;55(7):963-7.

52. Cornaggia CM, Beghi M, Moltrasio L, Beghi E, RESt-1 Group. Accidents at work among people with epilepsy: results of a European prospective cohort study. Seizure. 2006;15(5):313-9. 